\title{
I.B.S. coatings on large substrates: Towards an improvement of the mechanical and optical performances
}

\author{
D. Forest, P. Ganau, B. Lagrange, J.M. Mackowski, C. Michel, J.L. Montorio, N. Morgado, R. Pignard, \\ L. Pinard, A. Remillieux \\ Laboratoire des Matériaux Avancés- VIRGO, Université Claude Bernard Lyon I, 22 Bd Niels Bohr - 69622 Villeurbanne Cedex France \\ name@ipnl.in2p3.fr
}

\begin{abstract}
Large mirrors $(\varnothing 350 \mathrm{~mm})$, having extremely low optical loss (absorption, scattering, wavefront) were coated for the VIRGO interferometer. The new mirror generation needs better wavefront and lower mechanical loss. The first results are discussed. (C)2004 Optical Society of America OCIS codes: (240.0310) Thin Films; (160.4670) Optical Material; (120.3180) Interferometry
\end{abstract}

\section{Introduction}

The experimental effort to detect gravitational waves is entering an important phase. A number of large-scale interferometers, such as VIRGO (Franco-Italian) or LIGO (American), are being built around the world and most should be operational in the next few years. All the subsets are now put together and VIRGO, for example, is ready for the commissioning phase. The aim is to reach the theoretical sensitivity curve as soon as possible by improving the different subsets.

Nevertheless, the sensitivity of interferometric gravitational waves observatories is limited by fundamental noise sources. In particular, the thermal noise of the suspended mirrors is expected to be the limiting noise source in the mid-frequency region $(50-500 \mathrm{~Hz})$, and especially the one associated with optical coatings. Unfortunately, this is the most interesting frequency band (many events per year) [1]. Knowing the mechanical quality factor $Q$ of the coated mirror gives a precise idea of its contribution to the thermal noise. The results obtained within the study we have started with LIGO (R\&D contract) will be described and some promising solutions are proposed to improve the mechanical losses of the IBS (Ion Beam Sputtering) thin films without damaging their optical properties.

The first generation of VIRGO mirrors we produced has remarkable optical performances at $1064 \mathrm{~nm}$ : absorption $<1$ ppm, scattering $<5 \mathrm{ppm}$, wavefront flatness around $3 \mathrm{~nm}$ RMS on $\varnothing 150 \mathrm{~mm}$. At this level, improving the absorption and the scattering becomes very difficult. But, by using the "corrective coating "technique [2], we are able to correct the wavefront substrate imperfections and to reach the " $1 \mathrm{~nm}$ RMS" specifications on large areas. The first results are discussed.

The "corrective coating" technique can also be applied to realize special shapes on substrates: classical ones like sphere or more complicated ones like the "Mexican Hat" (MH) profile. The MH usefulness will be described in the following paragraphs and the preliminary results will be described.

\section{Mechanical loss improvement.}

The large mirrors are made of ultra-pure silica substrates (Suprasil 311 or $312 \mathrm{SV}$ from HERAEUS) on which we have deposited a coating formed by IBS alternating layers of silicon dioxide $\left(\mathrm{SiO}_{2}\right)$ and tantalum pentoxide $\left(\mathrm{Ta}_{2} \mathrm{O}_{5}\right)$. This type of coatings was chosen because it can be highly reflective in a narrow band around $1064 \mathrm{~nm}$, while having very low absorption and scattering losses.

The effect of coatings on the mirror thermal noise was investigated only very recently [3] because some calculations indicated that the mechanical losses $\phi_{\mathrm{C}}$ of the coatings (intrinsic mechanical dissipation, inverse of the quality factor $\mathrm{Q}$ at the resonance frequency $\mathrm{f}_{0}$ ) could be much greater than expected. The total mechanical loss of an optical component is equal to the sum of the intrinsic loss of the substrate $\phi_{\mathrm{S}}$ plus any loss associated with the coating (equation (1), $\mathrm{E}_{\mathrm{C}} / \mathrm{E}_{\mathrm{S}}$ is the ratio of energy stored in the coating and in the substrate).

$$
\phi_{\text {Total }}\left(f_{0}\right) \approx \phi_{S}\left(f_{0}\right)+\frac{E_{C}}{E_{S}} \phi_{C}\left(f_{0}\right)
$$

We started this study with the LIGO community (R\&D contract), which makes the Q measurement. Experiments (excitation of the resonant modes of uncoated or coated samples) confirmed that multi-layers of $\mathrm{Ta}_{2} \mathrm{O}_{5} / \mathrm{SiO}_{2}$ add significant levels of mechanical loss, when applied to fused silica substrate [3]. Indeed, a micropolished substrate (diameter 3", thickness 0.25 or $2.5 \mathrm{~cm}$ ) has a Q factor of $\mathbf{1 0}^{7}$. The Q factor decreased to a value of $\mathbf{4} \mathbf{1 0}^{\mathbf{5}}$ on coated surface (this corresponds to a coating loss of $\mathbf{2 . 7} \mathbf{1 0}^{-4}$ ). This difference can be greater if the substrate is annealed 
before coating $\left(\mathrm{Q}_{\text {substrate }}=\mathbf{5} \mathbf{1 0}^{\mathbf{7}}\right)$ [4]. The annealing produces a substrate stress relaxation. The stress was created during the fused silica fabrication and the polishing phase.

To find the origin of the mechanical loss in the coatings, we decided to investigate three potential sources of mechanical loss, in collaboration with LIGO:

$>$ The coating-substrate interface

$>$ The interfaces between high and low index layers

$>$ The coating materials.

By depositing quarter-wave multi-layers $(\mathrm{HL})^{\mathrm{n}}$ at $1064 \mathrm{~nm}$ with different numbers of layers, we eliminated the first hypothesis as the coating loss increases with the number of doublets $\mathrm{n}$. The loss comes from the coatings, not from the coating-substrate interface.

In the same way, we have deposited two types of multi-layers: $(\mathbf{H L})^{\mathrm{n}}$ and $(\mathbf{H} / \mathbf{2} \mathbf{L} / \mathbf{2})^{\mathbf{2 n}}$. The total thickness for each layer is the same but the interface number is twice. The measured coating loss is not dependent on the number of coating interfaces, which confirms that the second hypothesis is also not justified.

At last, to test the last potential loss source, we have deposited multi-layers with variable proportions of $\mathrm{Ta}_{2} \mathrm{O}_{5}$ and $\mathrm{SiO}_{2}:(\mathbf{H} / \mathbf{2} \mathbf{3 L} / \mathbf{2})^{\mathbf{n}}$ and $(\mathbf{3 H} / \mathbf{2} \mathbf{L} / \mathbf{2})^{\mathbf{n}}$. It appeared that the loss is directly proportional to the $\mathrm{Ta}_{2} \mathrm{O}_{5}$ thickness. From equation (1) [4], it is possible to separate the loss contribution of each material: the loss factor is eight times higher for $\mathrm{Ta}_{2} \mathrm{O}_{5}\left(\phi_{\mathrm{Ta} 2 \mathrm{O} 5}=0.510^{-4}, \phi_{\mathrm{SiO} 2}=4.410^{-4}\right)$.

Consequently, we have started to investigate other high index coating materials that may give lower mechanical loss while retaining the optical properties (high reflectivity, low scatter, low absorption): ternary alloys, doped materials. The aim is to decrease the coating stress, create more stable links between the layer atoms to avoid the links to vibrate (covalent links for instance). A simple way to start is to dope the tantalum pentoxide.

The first results are very promising. With a $2 \%$ dopant concentration, we decrease the multi-layer mechanical loss by $\mathbf{3 7 \%}\left(\mathbf{1 . 7} \mathbf{1 0}^{-4}\right.$ instead of $\left.2.710^{-4}\right)$. But, at the same time, the extinction coefficient at $1064 \mathrm{~nm}$ of the doped $\mathrm{Ta}_{2} \mathrm{O}_{5}$ layer increases $\left(6.3+/-0.510^{-7}\right.$ instead of $\left.4.5+/-0.510^{-7}\right)$. The mirror absorption increases also from $0.8 \mathrm{ppm}$ to $1.3 \mathrm{ppm}$ at $1064 \mathrm{~nm}$. Consequently, a happy medium has to be found: improving the mechanical loss and not damaging too much the optical performances.

This is the first positive result obtained on this subject, according to the literature. The nature of the dopant and its atomic concentration has not yet been optimized, but, there is a lot to do to reach the requirement $\left(\phi_{\text {Coating }}=\mathbf{4} \mathbf{1 0}^{-5}\right)$. This work will be done within an R\&D contract we have signed with the "Advanced LIGO" program.

\section{Substrate wavefront control.}

The VIRGO large mirrors we coated have a wavefront flatness of $3 \mathrm{~nm}$ RMS on $\varnothing 150 \mathrm{~mm}$. This good result will not be sufficient for the future mirror generation (1 nm RMS on $\varnothing 150 \mathrm{~mm})$. Thanks to a very good layer thickness uniformity $(0.3 \%$ on $\varnothing 700 \mathrm{~mm})$, we found the silica substrate flatness to be the limiting factor (same wavefront before and after coating). One solution consists in correcting the substrate surface before coating by the "corrective coating" technique [2]. A thin silica layer is deposited through a small fixed mask on the surface only where it is necessary. The sample-holder is a very accurate robot, which can make any movement in a one meter circle.

In the past [5], the correction was done after coating. But, due to interferences in the multi-layer, the wavefront correction was not linear with the silica thickness deposited and was limited in amplitude (90 nm at $1064 \mathrm{~nm})$. This is not true if the correction is done directly on the substrate: $1 \mathrm{~nm}$ deposited leads to $1 \mathrm{~nm}$ wavefront variation.

The wavefronts are measured with an ADE Phase Shift interferometer at $1064 \mathrm{~nm}$ (pupil $\varnothing 150 \mathrm{~mm}$ ). The first thing to do was to check the reproducibility R of the metrology (RMS difference between two successive measurements). Two configurations were tested (one measurement consists in an average of 10 acquisitions):

$>$ Sample not moved between two measurements: $\mathbf{R}=\mathbf{0 , 0 6} \mathbf{~ n m ~ R M S ~}$

$>$ Sample moved between two measurements : $\quad \mathbf{R}=\mathbf{0 , 2 0} \mathbf{~ n m ~ R M S ~}$

These values are satisfactory because the eventual limitation of the method will not come from the metrology line. Figure 1 shows the wavefront correction result obtained on a VIRGO type substrate $(\varnothing 156 \mathrm{~mm}$, thickness $55 \mathrm{~mm}$, roughness $0.5 \AA \mathrm{RMS}$ ). The first results are very encouraging. The wavefront on $\varnothing 110 \mathrm{~mm}$ decreased from $3.3+/-$ $0.1 \mathrm{~nm}$ RMS (16 nm PV) to $\mathbf{0 . 9}+/-\mathbf{0 . 1} \mathbf{~ n m}$ RMS (10 nm PV). The defect (small hole) near the center is linked to a technical problem of the robot, which has been identified. It should be rapidly solved.

The "corrective coating" method is really powerful and this result is the proof that obtaining a $1 \mathrm{~nm}$ RMS wavefront for the next VIRGO generation is not utopian. We checked after correction that the roughness was not changed (always around $0.5 \AA$ RMS) and that the number of point defects on the surface had not increased. 

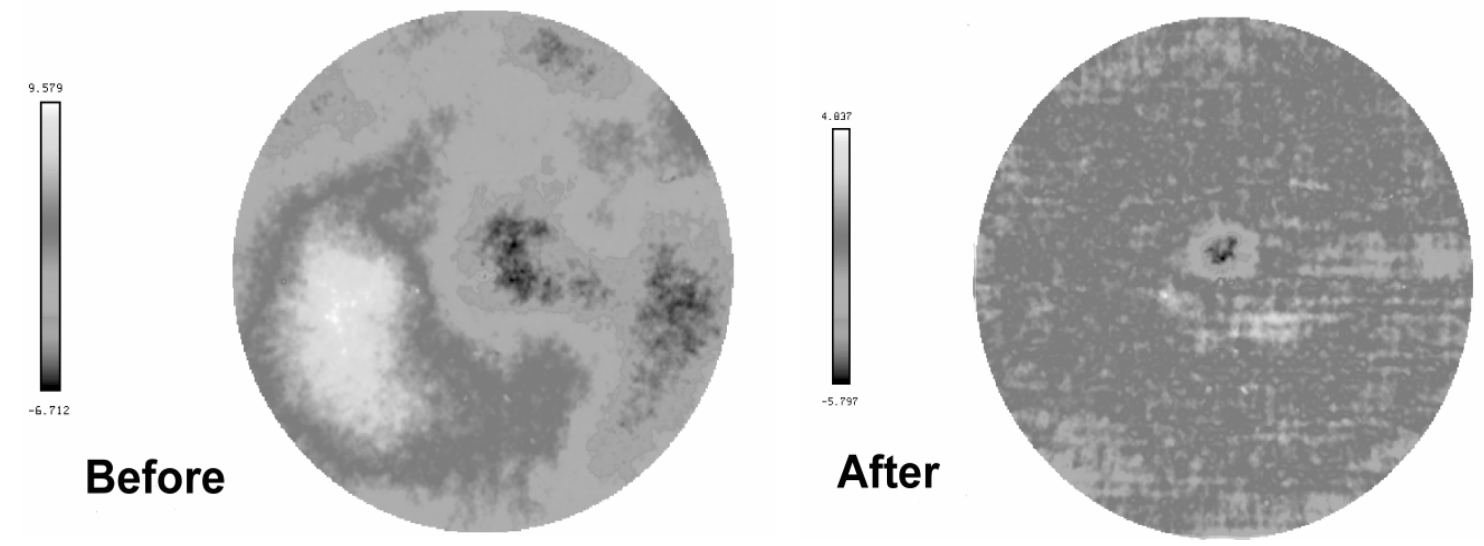

Figure 1: Wavefront correction of a micropolished substrate

Another important property of the "corrective coating" technique is to create special shapes on the substrate surface: for example a sphere or a "Mexican Hat" $(\mathrm{MH})$ profile. The aim of such complicated profile (Figure 2) is to use in the future generation of gravitational wave interferometers non-Gaussian (flat-topped) beams [6]. Thus, one can reduce the impact on the interferometer sensitivity of a variety of fundamental disturbances (thermoelastic noise, brownian thermal noise, coating thermal noise, thermal lensing). The laser beam energy produces a non uniform deformation of the mirror, due to the residual coating and substrate absorptions, which modify the average positioning of the mirror (the output phase shift of the interferometer is also modified). With cavity mirrors matching the phase profile of the flat-topped beam, the mirror heating will be more uniform and the interferometer sensitivity may be improved up to the standard quantum limit.
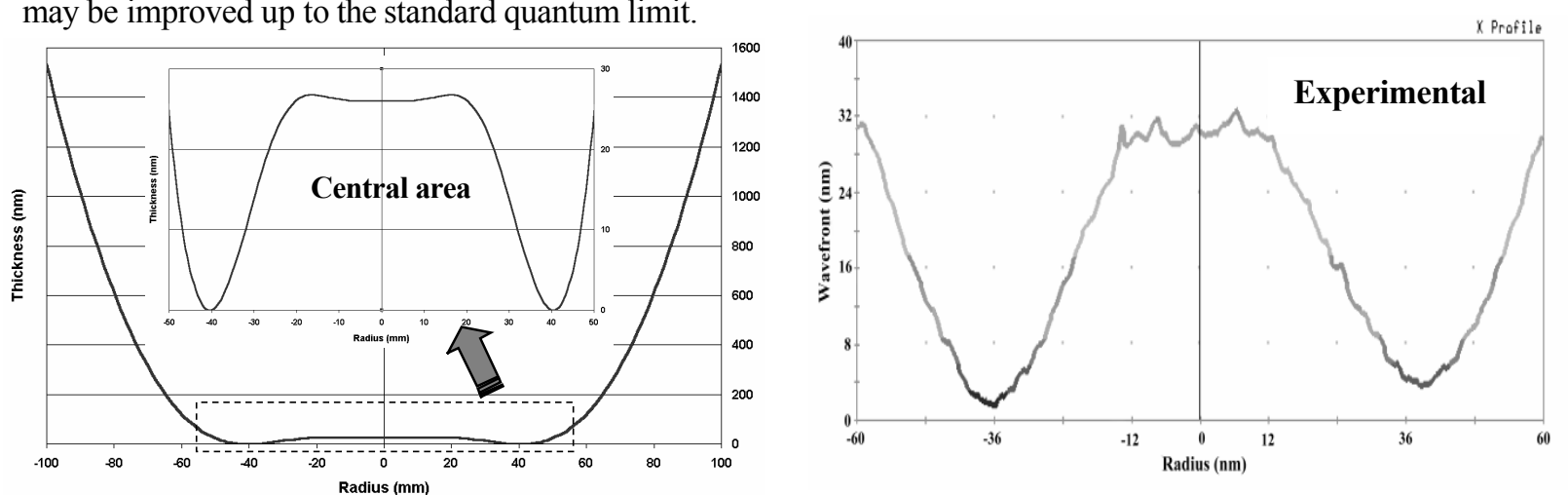

Figure 2: Theoretical and experimental profile of the "Mexican Hat" wavefront (central area)

Figure 2 displays the result of the first deposition of a MH profile on a VIRGO type silica substrate $(\varnothing 156 \mathrm{~mm})$. We have at first studied the central area of the profile, which is the most critical one (required accuracy close to $1 \mathrm{~nm}$ ). There is a good agreement between theory and experiment (same shape, same Peak to Valley level), but the flat zone near the center must be smoothed and widened.

\section{Conclusion}

Next mirror generation of large-scale interferometers needs IBS coatings having better optical and mechanical performances. Thus, we have started some R\&D studies (mechanical Q, substrate wavefront control) whose first results are very promising.

\section{References}

[1] P. Amico et al., "Mechanical quality factor of mirror substrates for VIRGO”, Class. Quantum Grav.19, 1663-1668 (2002).

[2] OSA: J.M. Mackowski, L. Pinard, L. Dognin, P. Ganau, B. Lagrange, C. Michel, M. Morgue, " Different approaches to improve the

wavefront of low-loss mirrors used in the VIRGO gravitational wave antenna”, Optical Interference Coatings, 1998 Vol.9, pp.MA7_1-MA7_3.

[3] G.M.Harry et al.," Thermal noise in interferometric gravitational wave detectors due to dielectric optical coatings", Class. Quantum Grav.19, 897-917 (2002).

[4] S.D. Penn et al., "Mechanical loss in Tantala/Silica dielectric mirror coatings", Class. Quantum Grav.20, 2917-2928 (2003).

[5] J.M. Mackowski, L. Pinard, L. Dognin, P. Ganau, B. Lagrange, C. Michel, M. Morgue, "VIRGO mirrors : Wavefront control ", Optical and Quantum Electronics Vol.31, n5/7 507-514 (1999)

[6] E. D'Ambrosio et al., "Status report on Mexican-Hat flat topped beams for advanced LIGO”, LIGO report n ${ }^{\circ}$ LIGO-T030009-00-R 